\title{
Structural origins of altered spectroscopic properties upon ligand binding in proteins containing a fluorescent non- canonical amino acid.
}

\author{
Patrick R. Gleason, ${ }^{1,2}$ Bethany Kolbaba-Kartchner, ${ }^{1,2}$ J. Nathan Henderson, ${ }^{1}$ Erik P. Stahl ${ }^{1,2}$, \\ Chad R. Simmons, ${ }^{1}$ and Jeremy H. Mills ${ }^{1,2}$ *
}

${ }^{1}$ The Biodesign Center for Molecular Design and Biomimetics, Arizona State University, Tempe, AZ, 85287, USA.

${ }^{2}$ School of Molecular Sciences, Arizona State University, Tempe, AZ, 85287, USA.

\section{Experimental Procedures}

\section{Protein Expression \& Purification}

Wild-type core streptavidin ${ }^{1}$ (SAV) and DNA oligonucleotides for making each Amber stop codon mutation were ordered from Integrated DNA Technologies, Inc. The gene encoding streptavidin was cloned into a pET29b vector using Gibson assembly. Mutations were made via overlap extension PCR and Gibson assembly. E. coli BL21 Star (DE3) cells were transformed with both a pEVOL plasmid ${ }^{2}$ containing a chloramphenicol resistance marker, two copies of the evolved CouRS synthetase, and an evolved tRNA specific to the evolved CouRS and a pET29 plasmid containing a kanamycin resistance marker and genes encoding the mutant streptavidins.

A single colony was used to inoculate $5 \mathrm{~mL}$ of $2 x Y T$ media, which was grown overnight in a shaking incubator at $37^{\circ} \mathrm{C}$ with 250 r.p.m. agitation until an $\mathrm{OD}_{600}$ of $\sim 5.0$ was reached. The cells were centrifuged for 10 minutes at $4200 \mathrm{x} g$ and resuspended in fresh $2 \mathrm{xYT}$ media. Arabinose $(0.2 \% \mathrm{w} / \mathrm{v})$ and the non-canonical amino acid, 7 -HCAA $(1 \mathrm{mM})$, were added to each culture and incubated for 1 hour at $37^{\circ} \mathrm{C}$ with 250 r.p.m. agitation in order to express and activate the CouRS synthetase. Isopropyl- $\beta$-Dthiogalactoside (IPTG) was then added to a final concentration of $1 \mathrm{mM}$ in order to induce expression of the SAV mutants. The cultures were then incubated between 8 and 16 hours at $30^{\circ} \mathrm{C}$, with 180 r.p.m. shaking. Cells were harvested via centrifugation and lysed in cell lysis buffer (25 mM Tris- $\mathrm{HCl}, 0.1 \%$ Triton-X 100, $3 \mathrm{mM} \beta$-mercaptoethanol). Inclusion bodies were collected via centrifugation at $20,000 \mathrm{xg}$ for 30 minutes and washed 3-4 times with wash buffer $(25 \mathrm{mM}$ Tris- $\mathrm{HCl}, 150 \mathrm{mM} \mathrm{NaCl}, 0.1 \%$ Triton-X 100). The washed

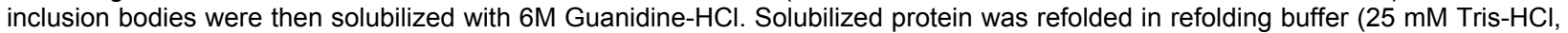
$20 \mathrm{mM}$ Imidazole, $500 \mathrm{mM} \mathrm{NaCl}$ ) by adding the protein solution dropwise to the fastest part of a rapidly spinning solution of refolding buffer. $^{3}$

The streptavidin mutants contained a C-terminal $6 x$ His-tag that allowed purification on a nickel-NTA resin (HisTrap FF, GE Healthcare). After loading onto the column, contaminant proteins were removed by flowing five column volumes (CV) of Ni-NTA wash buffer (25 mM Tris- $\mathrm{HCl}$ pH 8.0, $20 \mathrm{mM}$ Imidazole, $500 \mathrm{mM} \mathrm{NaCl}$ ) over the column followed by elution of SAV with five CV Ni-NTA elution buffer (25 mM Tris-HCl pH 8.0, $500 \mathrm{mM}$ Imidazole, $150 \mathrm{mM} \mathrm{NaCl})$. Fluorescent fractions were collected, combined, diluted 10x in IEC wash buffer $(25 \mathrm{mM}$ Tris- $\mathrm{HCl} \mathrm{pH} 8.0,10 \mathrm{mM} \mathrm{NaCl})$ and purified further on an anion exchange column (HiTrap Q FF, GE Healthcare). The column was washed with five CV of IEC wash buffer followed by a mixture of $90 \%$ wash buffer and $10 \%$ elution buffer (25 mM Tris-HCl pH 8.0, $500 \mathrm{mM} \mathrm{NaCl})$. Proteins were then eluted with five CV of $100 \%$ IEC elution buffer. Again, fluorescent fractions from the IEC purification were collected and combined. The combined fractions were then concentrated using a $10 \mathrm{kDa}$ molecular weight cutoff spin column (Amicon) to $500 \mu \mathrm{l}$. Concetrated protein was injected onto a size-exclusion column (Superdex 200 increase 10/300 gl, GE Healthcare) and eluted using Sizing Buffer (25 mM Tris-HCl pH 7.0, $150 \mathrm{mM} \mathrm{NaCl})$. Fractions that were fluorescent were collected, consolidated, and concentrated to a volume of $\sim 1 \mathrm{~mL}$ on a $10 \mathrm{kDa}$ molecular cutoff spin column (Amicon). Fractions were then refrigerated at $4{ }^{\circ} \mathrm{C}$ for use in experimental procedures.

\section{SDS-PAGE analysis of mutants}

Single colonies of $E$. coli harboring each SAV mutant were used to inoculate $5 \mathrm{~mL}$ cultures of 2xYT medium supplemented with $50 \mu \mathrm{g} / \mathrm{mL}$ kanamycin and $34 \mu \mathrm{g} / \mathrm{mL}$ chloramphenicol. Cells were grown overnight at $37^{\circ} \mathrm{C}$ with 250 r.p.m. shaking. The cultures were then centrifuged at $4,200 \mathrm{xg}$. and the media was decanted. Each pellet was resuspended in $5 \mathrm{~mL}$ of fresh $2 \mathrm{xYT}$ media containing $0.2 \%$ arabinose, $50 \mu \mathrm{g} / \mathrm{mL}$ kanamycin and $34 \mu \mathrm{g} / \mathrm{mL}$ chloramphenicol. The cultures were divided equally and 7-HCAA (1 mM) was added to only one of the two cultures. Cultures were then incubated at $37^{\circ} \mathrm{C}$ and 250 r.p.m. shaking for 1 hour. IPTG was then added to a final concentration of $1 \mathrm{mM}$ and protein expression was allowed to continue at $30^{\circ} \mathrm{C}$ with 225 r.p.m. shaking for 16 hours. $15 \mu l$ of each culture or $1 \mu \mathrm{g}$ of commercially available Streptavidin (Sigma) were mixed with $15 \mu \mathrm{l} 4 \mathrm{x}$ Laemmli loading buffer and was incubated at $95^{\circ} \mathrm{C}$ for 10 minutes followed by $25^{\circ} \mathrm{C}$ for 5 minutes. $15 \mu \mathrm{l}$ of each sample was loaded into each well of a $4 \%$ stacking $/$ $15 \%$ resolving polyacrylamide gel and subjected to $125 \mathrm{~V}$ for 90 minutes. 


\section{Mass spectrometry}

Streptavidin variants were expressed and purified as described above and were buffer exchanged into $18 \mathrm{M} \Omega$ water prior to mass spectrometric analysis. Data were collected via direct injection on an Agilent 6530 Accurate-Mass Q-TOF LC/MS instrument. Deconvolution of the spectra was carried out with the Agilent Masshunter Bioconfirm software package.

\section{Western Blotting}

An acrylamide gel was equilibrated in $25 \mathrm{~mL}$ of transfer buffer $(25 \mathrm{mM}$ Tris, $\mathrm{pH}$ 8.3, $192 \mathrm{mM}$ glycine, $20 \%$ methanol, $0.1 \%$ SDS) for 60 minutes. Protein from the acrylamide gel was transferred to a nitrocellulose membrane using a semi-dry transfer apparatus (Bio-Rad) until the pre-stained ladder (Precision Plus Protein, New England Biolabs) was completely transferred to the membrane (30 minutes at $25 \mathrm{~V})$. The membrane was removed from the apparatus and incubated with a TBS-T blocking solution ( $25 \mathrm{mM}$ Tris, $\mathrm{pH}$ 8.0, $150 \mathrm{mM} \mathrm{NaCl}, 0.1 \% \mathrm{v} / \mathrm{v}$ Tween-20, $5 \% \mathrm{w} / \mathrm{v}$ powdered nonfat milk) for 60 minutes. The membrane was then washed 3 times for 5 minutes with $25 \mathrm{~mL}$ of TBS-T (25 mM Tris, $\mathrm{pH} 8.0,150 \mathrm{mM} \mathrm{NaCl}, 0.1 \% \mathrm{v} / \mathrm{v}$ Tween-20) on an orbital shaker, followed by incubation in TBS-T with an HRP-conjugated anti-streptavidin antibodies (Abcam; 1:10000 dilution) at room temperature for 1 hour on an orbital shaker. The membrane was then washed with $25 \mathrm{~mL}$ TBS-T 3 times for 5 minutes each on an orbital shaker. A 1x solution of DAB solution (Pierce) was created immediately before use by adding $2.5 \mathrm{~mL}$ of $10 x$ DAB with $22.5 \mathrm{~mL}$ of Stable Peroxide Substrate Buffer. Finally, the membranes were incubated for 30 minutes in the 1x DAB solution, air-dried, and imaged.

\section{Biotin Dependent Response}

$10 \mu \mathrm{L}$ of $10 \mathrm{mM}$ biotin was serial diluted in TBS ( $25 \mathrm{mM}$ Tris- $\mathrm{HCl} \mathrm{pH} \mathrm{7.0,150} \mathrm{mM} \mathrm{NaCl})$ to concentrations of $1 \mathrm{~mm}, 100 \mu \mathrm{M}$, $10 \mu \mathrm{M}, 1 \mu \mathrm{M}, 100 \mathrm{nM}, 10 \mathrm{nM}, 1 \mathrm{nM}$, and $100 \mathrm{pM}$. $10 \mu \mathrm{L}$ of each biotin concentration was then mixed with $90 \mu \mathrm{L}$ of SAV mutant (10 $\mu \mathrm{M}$ ) in TBS to give concentrations of: $100 \mu \mathrm{M}, 10 \mu \mathrm{M}, 1 \mu \mathrm{M}, 100 \mathrm{nM}, 10 \mathrm{nM}, 1 \mathrm{nM}, 100 \mathrm{pM}$ and $10 \mathrm{pM}$. Samples were excited at 340 $\mathrm{nm}$ and fluorescence was measured at $450 \mathrm{~nm}$ using a $1 \mathrm{~cm}$ quartz cuvette (Starna Cells; Atascadero, CA) on Horiba Nanolog fluorimeter. All data were normalized to the highest value and are the average of three readings. Curve fitting was performed with the Prism 9 software package (Graphpad; San Diego, CA) using the 4-parameter logistic (4PL) non-linear regression function (Eq. 1). $K_{d}$ values derived from these fits are listed in Table S1.

$$
f(x)=d+\frac{a-d}{1+\left(\begin{array}{l}
x \\
c
\end{array}\right)}
$$

\section{Supplemental analysis of SAV mutant spectra}

\section{L25X Characterization}

The L25X mutant (where $X$ signifies mutation of the native residue to $7-$ HCAA) shows a $40 \%$ increase in emission at 450 $\mathrm{nm}$ in the presence of biotin (Table 1, Figure 2b). A single absorbance maximum at $325 \mathrm{~nm}$ was observed for the L25X mutant in both the apo and holo forms (Figure $2 \mathrm{~b}$ inset), which suggests that 7-HCAA exists primarily in the neutral form irrespective of the presence of biotin. Structural characterization of this mutant was precluded by low protein expression yields. However, the fact that the absorbance spectra of the apo and bound forms are nearly identical suggests that the chemical environment surrounding the 7-HC phenol in this mutant is not substantially altered by the addition of biotin.

In an effort to gain additional insight into the orientation adopted by 7-HCAA in this mutant, we used the PyMOL molecular viewing software ${ }^{4}$ to mutate residue L25 to 7-HCAA in the apo form of SAV (PDB ID: 1swc). In this analysis, the ethylene linker of 7HCAA was fit to the existing rotamer of the native leucine residue up to the $y$-carbon (Figure S3) and rotation around $x 2$ and $x 3$ was sampled. Although we cannot be certain that 7-HCAA adopts this rotamer in the L25X mutant, this placement immediately suggested a number of residues with which 7-HCAA could interact in ways that are consistent with the spectroscopic data. First, a hydrogen bond could be formed between the 7-HCAA phenol and residue S45 (Figure S3a). Another possibility is the formation of hydrogen bonds between the 7-HCAA phenol and residues Y43 and D128 (Figure S3b). Hydrogen bonding interactions between 7-HCAA and surrounding residues have been suggested as a potential quenching mechanism in a previous study. ${ }^{5}$ If any of these interactions were to form, it is likely that they would be disrupted upon biotin binding, which could give rise to the observed increase in fluorescence in the holo form of this protein. Although purely conjectural, the analysis above highlights residues that may serve to quench 7-HCAA fluorescence when in proximity to the fNCAA.

\section{L124X characterization}

Both the absorbance and emission spectra of the L124X mutant exhibit features distinct from those observed in all other variants. The absorbance spectra of both apo and holo L124X are essentially linear from $300 \mathrm{~nm}$ to $450 \mathrm{~nm}$. The emission spectrum of this mutant exhibits a major peak at $450 \mathrm{~nm}$ with a significant shoulder at $380 \mathrm{~nm}$. The substantial emission at $380 \mathrm{~nm}$ suggests that that ESPT from 7-HCAA could be at least partially blocked in this mutant. Although increases in emission intensity are observed at both $380 \mathrm{~nm}$ and $450 \mathrm{~nm}$ upon biotin binding, the magnitude of this change is substantially smaller than those observed for all other variants (Table 1, Figure 2). Unfortunately, L124X suffered from low initial expression yields and exhibited consistent decreases in yield throughout each stage of the purification process; characterization of this protein was limited to spectroscopic analysis alone. In an effort to better understand these data, we modeled 7-HCAA in place of residue 124 in the wild type apo structure of SAV (PDB ID: 1swc) using the PyMOL software. We immediately recognized that position 124 is directly adjacent to the identical residue in a symmetry related subunit; the $C_{\alpha}-C_{\alpha}$ distance between these residues is only $5.0 \AA$. Without structural data, it is difficult to confidently predict the orientations adopted by the two 7- HC moieties in this protein. However, given the close proximity of the sites of incorporation, the possibility exists that the two fluorophores would directly interact, which could potentially lead to the unique spectra we observed. 


\section{Supplemental Schemes and Figures}

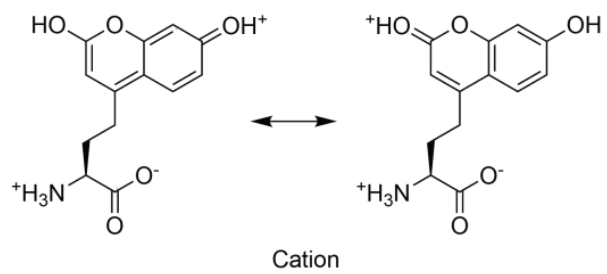

(ex. 325 nm / em. 420 nm)

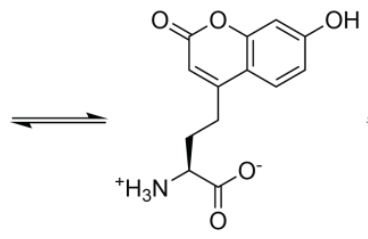

Keto Tautomer (ex. $325 \mathrm{~nm} /$ em. $380 \mathrm{~nm}$ )

1<smiles>[NH3+]C(CCc1cc(O)oc2cc(=O)ccc1-2)C(=O)[O-]</smiles>

Keto Tautomer (ex. 325 nm / em. 475 nm)

Scheme S1. Tautomeric and ionized forms of L-(7-hydroxycoumarin-4-yl)ethylglycine and their excitation/emission wavelengths. Values are from small molecule studies performed in aqueous solutions. ${ }^{6}$

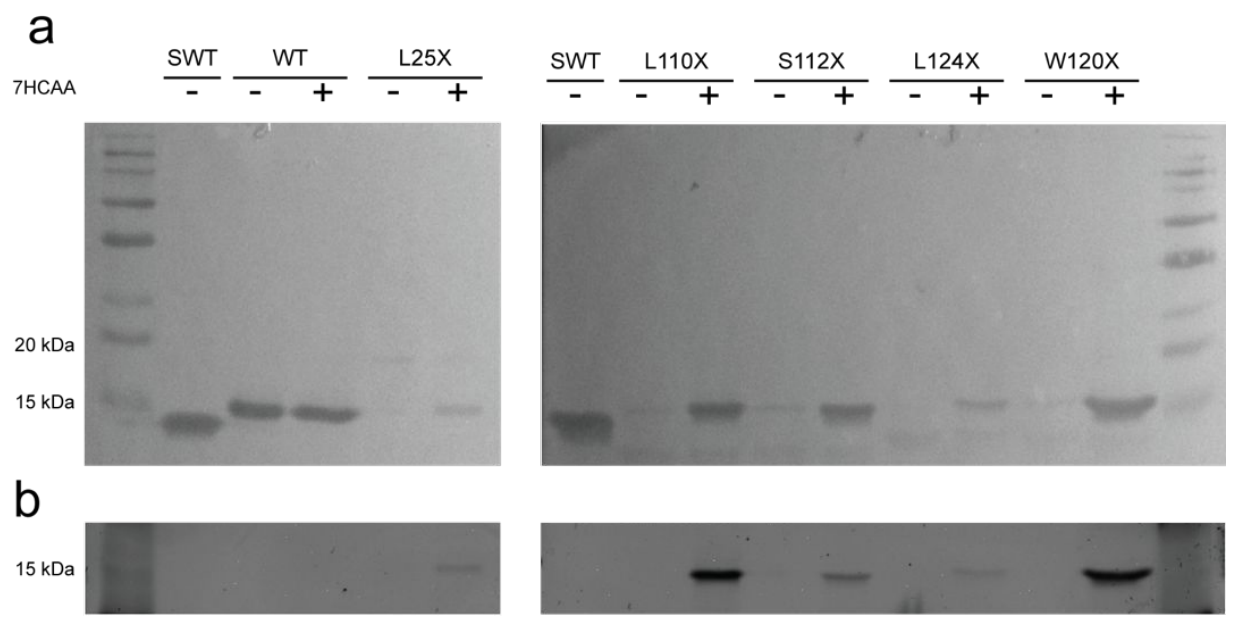

Figure S2. Streptavidin mutant expression. (a) Western blot and (b) fluorescent images of whole cell Escherichia coli lysates for each streptavidin mutant. Each mutant was expressed in both the presence (+) or absence (-) of 7-HCAA. $1 \mu \mathrm{g}$ of commercially purchased wild-type streptavidin (SWT) without a 6x-his tag was used as a standard. Faint bands can be detected in the L25X, L110X, S112X, L124X, and W120X lanes without 7-HCAA, which is likely a consequence of amber codon suppression with tyrosine in lieu of 7-HCAA. Faint bands below each of the full-length L110X, S112X, L124X, and W120X bands are likely a consequence of truncated protein in which the amber stop codon was not fully suppressed. 

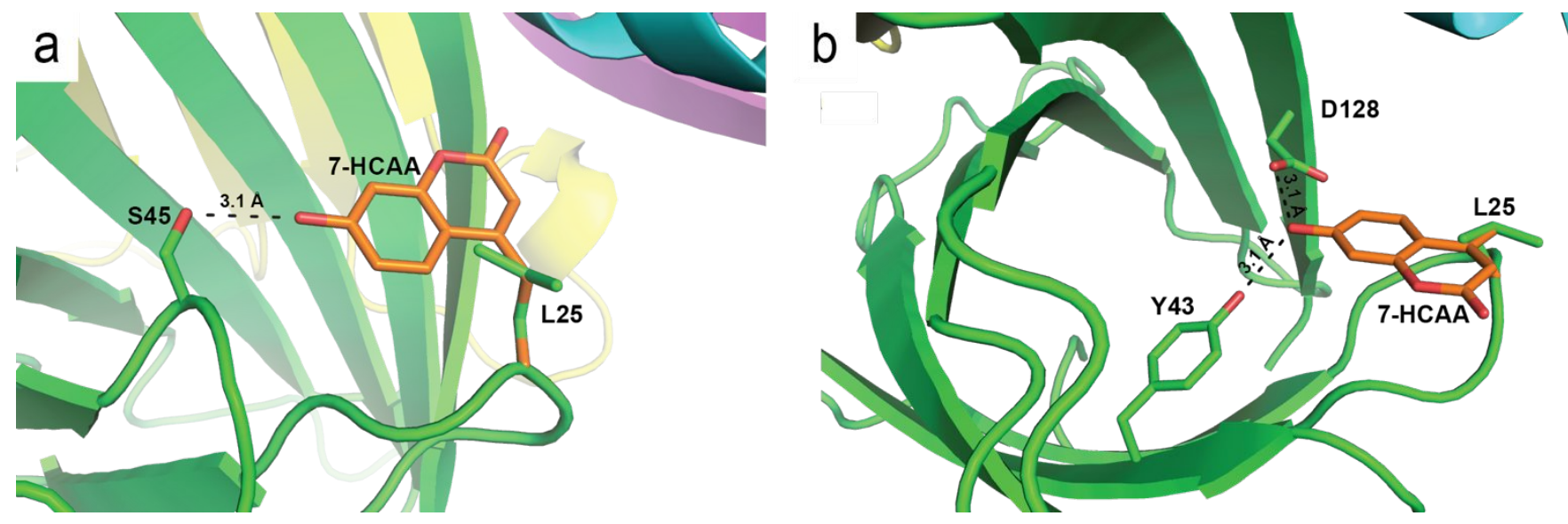

Figure S3. Potential interactions between 7-HCAA and surrounding residues in the L25X mutant. 7-HCAA was overlaid on residue L25 using the PyMOL molecular viewing software by fitting the $\mathrm{Ca}, \mathrm{C} \beta$, and $\mathrm{C}_{Y}$ atoms of 7-HCAA to the identical atoms in the native leucine. Rotation about $X_{2}$ and $X_{3}$ was carried out in order to simulate conformational sampling that might occur in the mutant protein. A number of potential interactions with surrounding residues were observed, name (a) residue S45 and (b) residues Y43 and D128 in the binding pocket of apo SAV. 


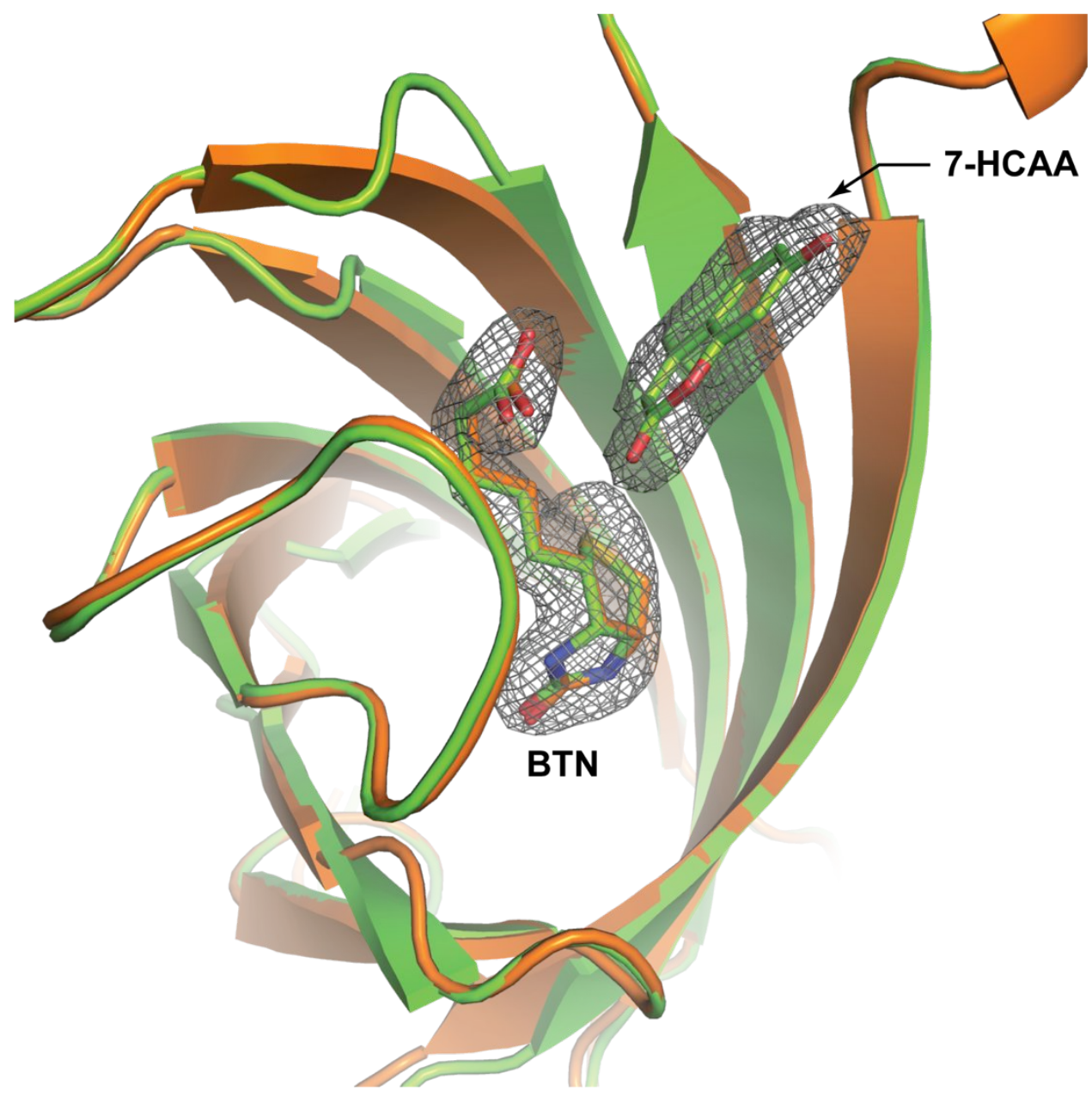

Figure S4. Comparison of biotin (BTN) binding in L110X and wt SAV. A superimposition of the holo form of the L110X mutant (green) with chain A of wild type, biotin-bound SAV (orange, PDB ID: 3ry2) is shown. All electron density displayed in this figure derives from the L110X crystal structure and is shown as a $2 F_{O}-F_{C}$ map contoured to $1 \sigma$. Biotin molecules from the L110X mutant and wild type SAV are shown in green and orange sticks, respectively. Structures were aligned using the super command in PyMOL. The RMSD of the biotin in the L110X crystal structure to the biotin in 3ry2 was calculated to be $0.149 \AA$ using the rms_cur command in PyMOL, which suggests that biotin adopts its native binding orientation in this streptavidin variant. 

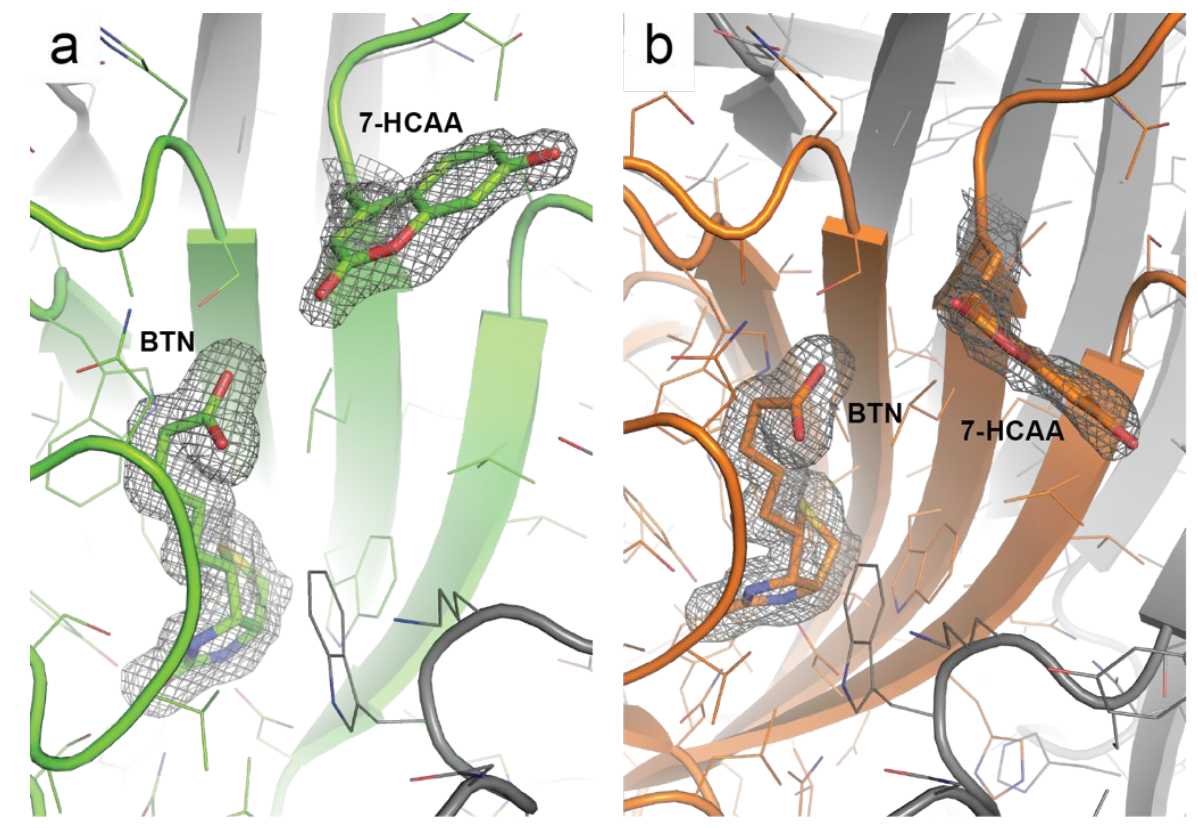

Figure S5. Structures of S112X containing biotin (BTN). The orientations adopted by 7-HCAA differ between subunits in this structure. (a) Chain A (green) and (b) chain B (orange) of the asymmetric unit are shown. Symmetry related chains are colored in grey. Electron density around each residue is shown as a $2 \mathrm{~F}_{\mathrm{O}}-\mathrm{F}_{\mathrm{C}}$ map contoured to $1 \sigma$. 


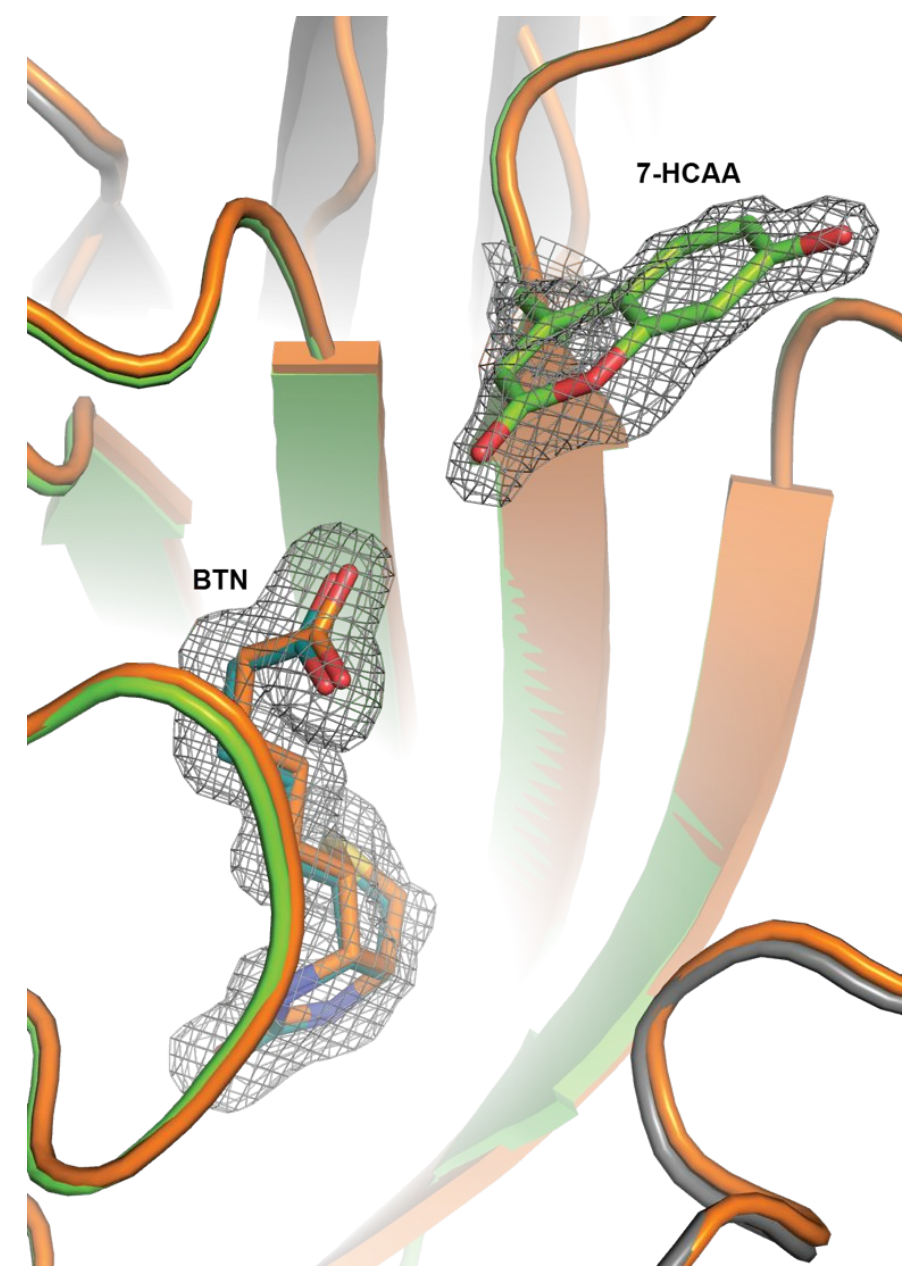

Figure S6. The structure of the biotin (BTN) bound S112X mutant (green) is superimposed with biotin bound wild type SAV (orange, PDB ID: 3ry2). Structures were aligned using the super command in PyMOL. The RMSD of the biotin in the S112X crystal structure to the biotin in 3ry2 was calculated to be $0.275 \AA$ using the rms_cur command in PyMOL. Electron density around the $\mathrm{S} 112 \mathrm{X}$ biotin is shown as a $2 \mathrm{~F}_{\mathrm{O}}-\mathrm{F}_{\mathrm{C}}$ map contoured to $1 \mathrm{\sigma}$. 


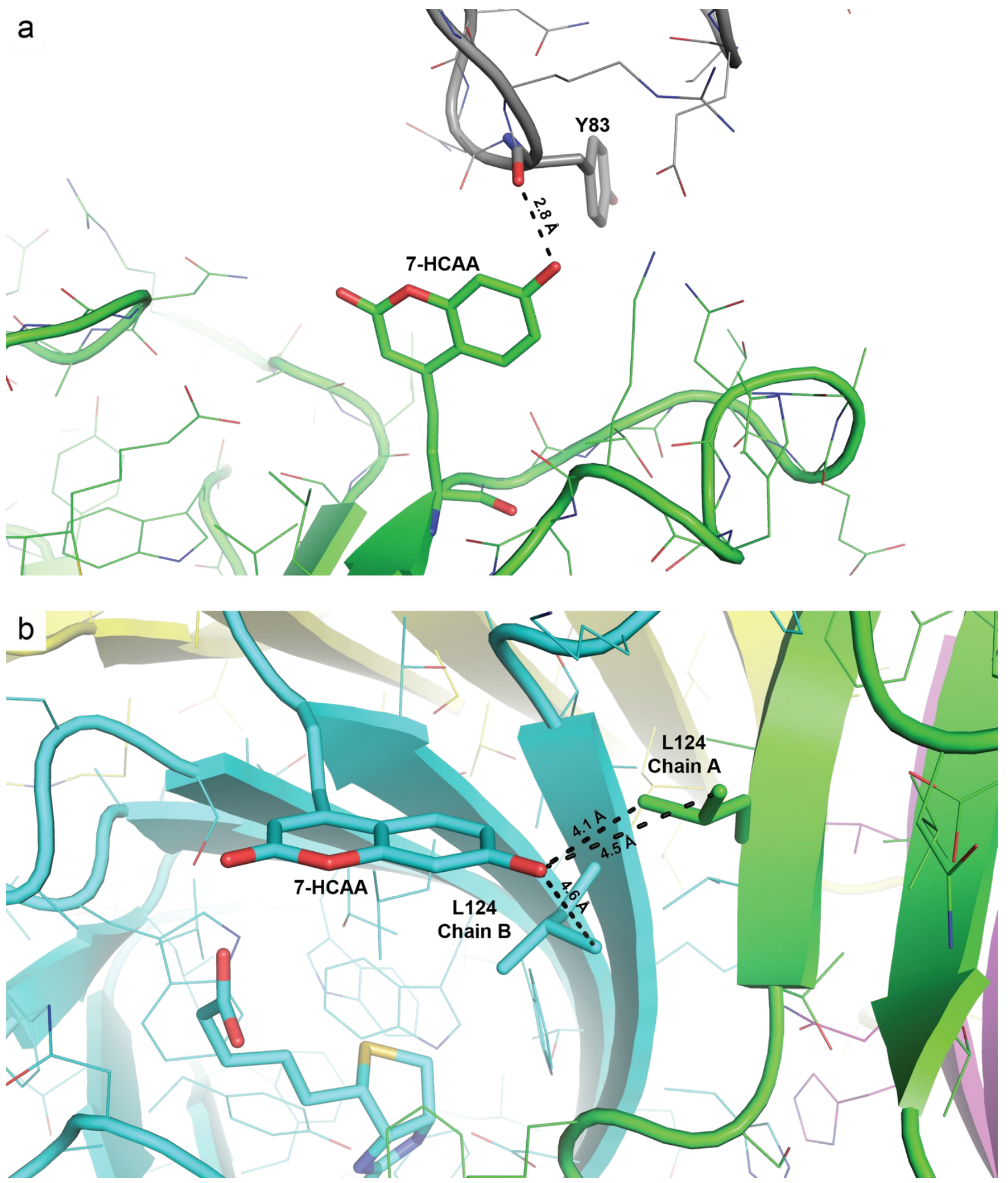

Figure S7. Crystal structures of the S112X mutant showing interactions between the 7-HCAA side and surrounding residues. Two conformations of 7HCAA were observed in chains A (a) and B (b) of this structure. Chain A (a) shows a hydrogen bond between the 7-HCAA and the Y83 of a subunit in an adjacent crystal. In chain B (b), 7-HCAA's phenol is found in in a more hydrophobic environment created by symmetry-related L124 residues in chains A and B (panel B). Dashed black lines illustrate distances between the 7-HCAA phenol and atoms on near neighbor residues; they are not indicative of hydrogen bonds. 


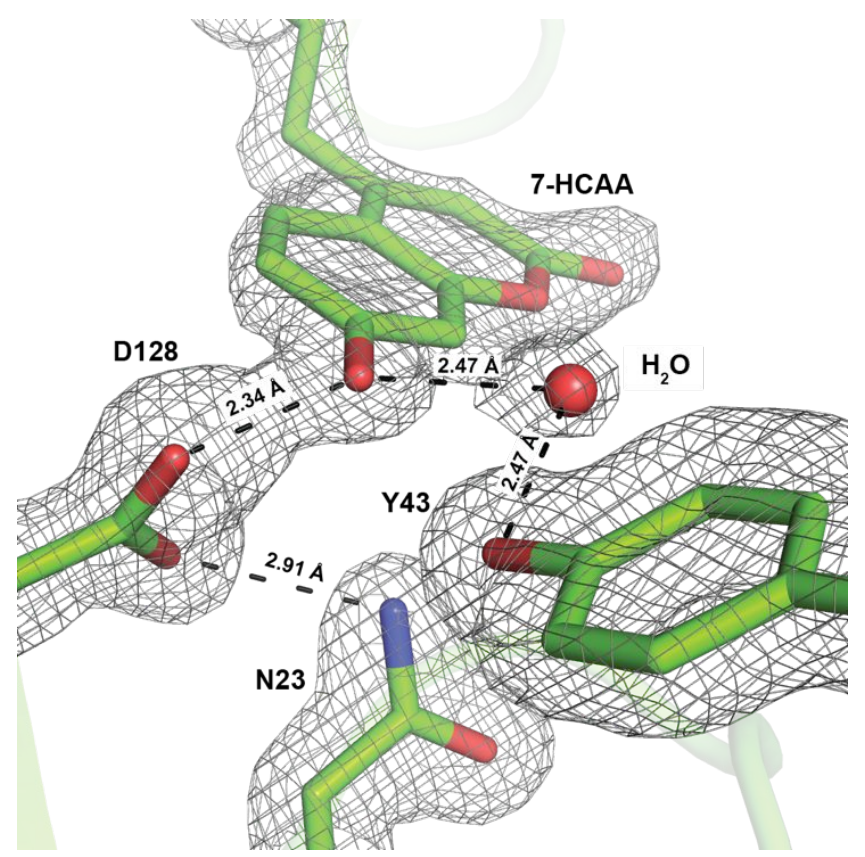

Figure S8. An apparent hydrogen bonding network in the W120X mutant. Distances are the averages of the two monomeric streptavidin chains in the asymmetric unit. Electron density around each residue is shown as a $2 F_{\mathrm{O}}-\mathrm{F}_{\mathrm{C}}$ map contoured to $1 \sigma$.

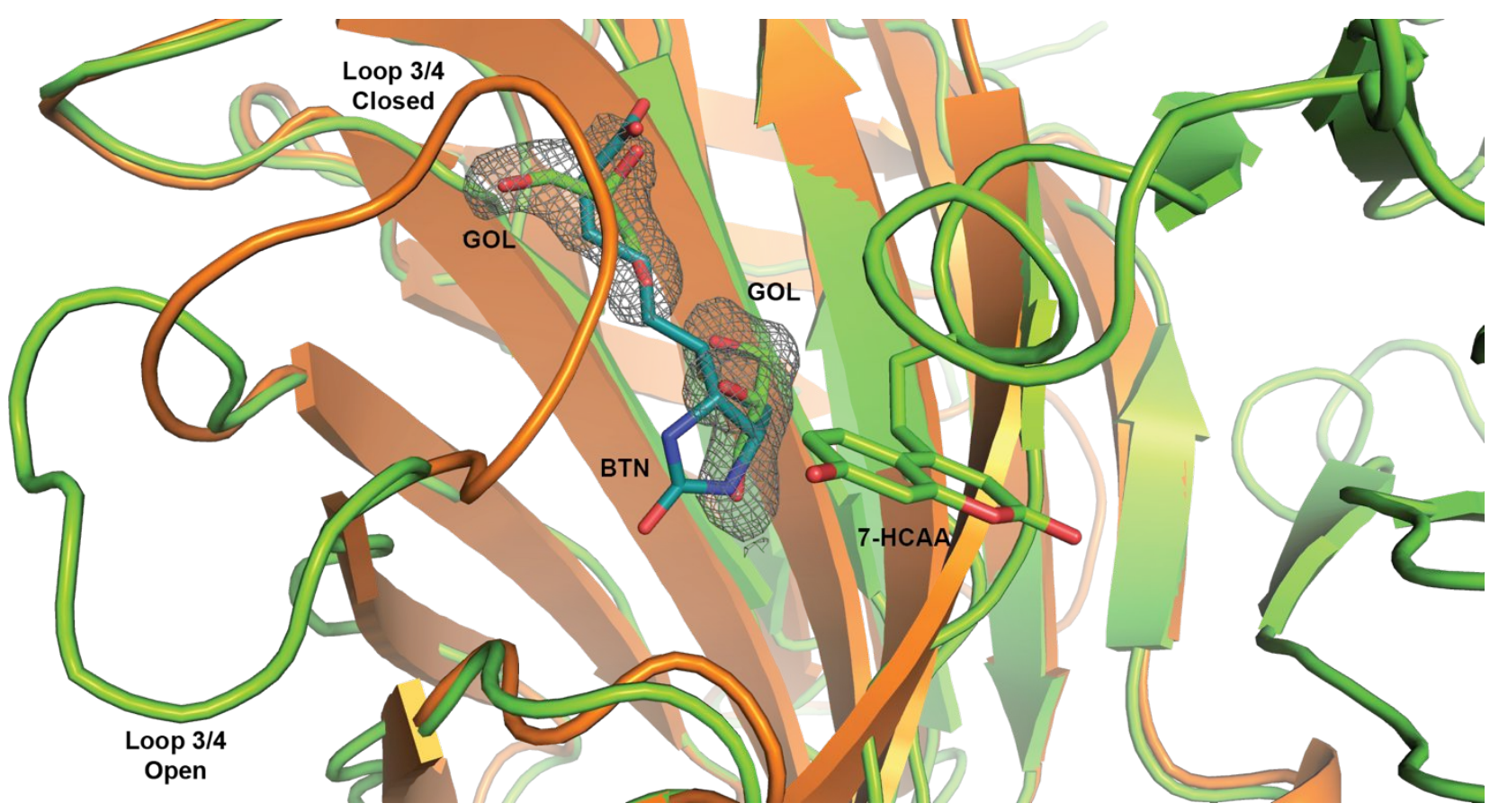

Figure S9. A crystal structure of W120X solved when glycerol was used as a cryoprotectant (green) superimposed on a biotin (BTN; teal sticks) bound SAV structure (PDB 3ry2, orange). The bound glycerol (GOL; green sticks) molecules in our structure occupy the biotin binding pocket. Loop 3/4 is also observed in the open position in the W120X crystal structure. The open position of this loop may lead to the observed differences between the W120X + biotin and W120X + glycerol fluorescence spectra shown in figure S10. It is also possible that the biotin itself contributes to these differences. Electron density around each glycerol molecule is shown as a 2FO-FC map contoured to $1 \sigma$. 


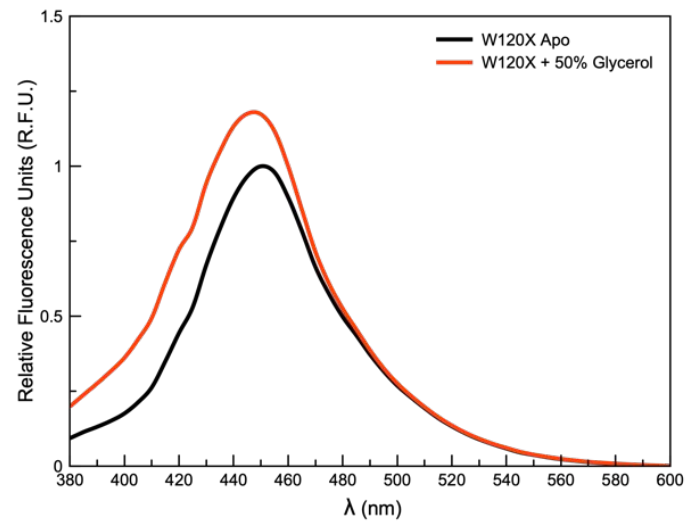

Figure S10. A normalized fluorescence spectrum of W120X in the absence (black) and in the presence (red) of glycerol. The protein concentration was $\sim 10 \mathrm{uM}$ and glycerol was present at $50 \%$. An excitation wavelength of $340 \mathrm{~nm}$ was used. 
a
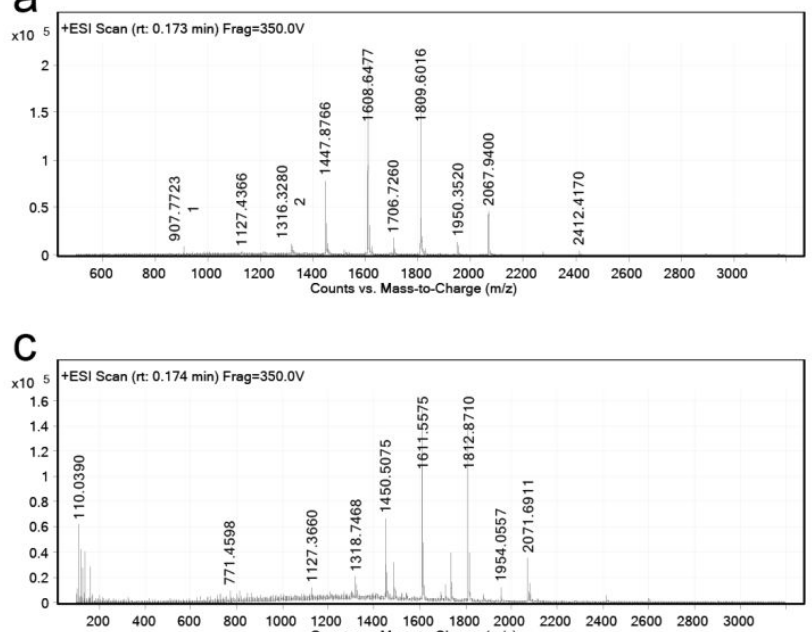

$\begin{array}{lllllllllllll}200 & 400 & 600 & 800 & 1000 & \begin{array}{l}1200 \\ \text { Counts vs. Mass-to-Charge }(\mathrm{m} / \mathrm{z})\end{array} & 2200 & 2400 & 2600 & 2800 & 3000\end{array}$

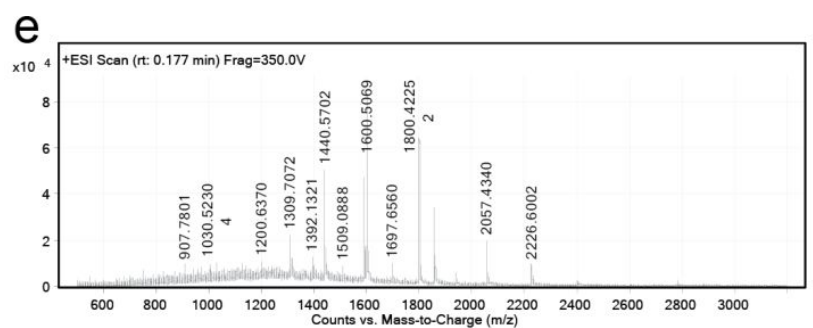

b

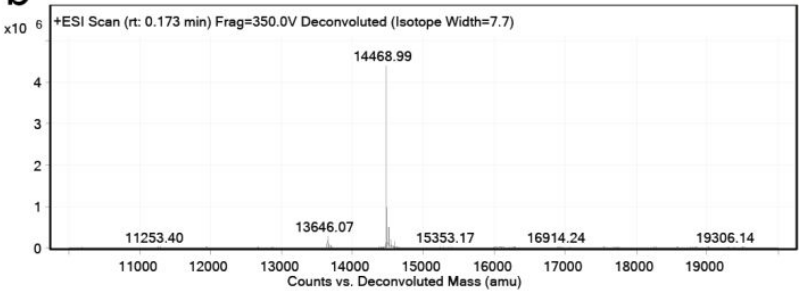

d

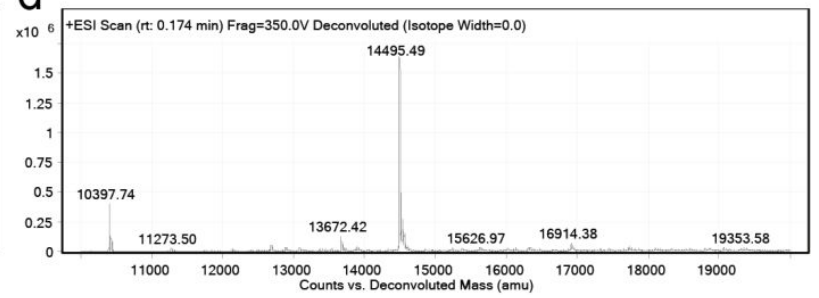

f

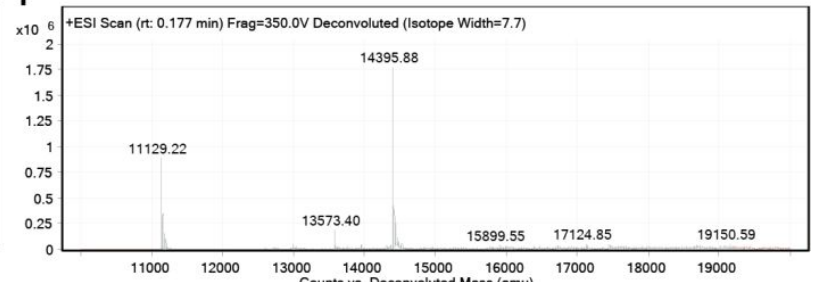

Figure S11. Mass spectrometric analysis of mutant streptavidins. Raw and deconvoluted mass spectra are shown for the L110X (panels a and $b$, respectively), S112X (panels $c$ and d, respectively) and W120X (panels e and $f$, respectively) SAV variants. Because the evolved tRNA/aminoacyl tRNA synthetase pair that was used to incorporate 7-HCAA in this study has been previously observed to occasionally incorporate tyrosine in response to the amber codon ${ }^{5}$ we collected these data in an effort to establish the efficiency of 7-HCAA incorporation in our mutant proteins. Our initial studies suggested that the $\mathrm{N}$-terminal methionine residue had been cleaved in each of our proteins. Expected and observed masses for each variant (without the $\mathrm{N}$-terminal Met) were: L110X expected: 14669.46 Da, observed: 14668.99 Da; S112X expected: 14495.54 Da, observed: 14495.49 Da; W120X expected: 14396.41 Da, observed: $14395.88 \mathrm{Da}$. All observed masses are within one Dalton of the expected values. Importantly, no peaks corresponding to tyrosinecontaining variants of these mutants (expected masses of tyrosine-containing variants: L110X: 14387.41 Da, S112X: 14413.49 Da, W120X: 14314.35 Da) were observed. Expected masses were calculated using Protein Prospector (https://prospector.ucsf.edu/prospector/mshome.htm) and Molecular Mass Calculator (https://www.Ifd.uci.edu/ gohlke/molmass/). 
Table S1. Parameters and statistics for L110X, S112X, and W120X apparent $K_{d}$ curve fits

\begin{tabular}{|c|c|c|c|}
\hline Construct & L110X & S112X & W120X \\
\hline \multicolumn{4}{|c|}{ Best-fit values } \\
\hline Bottom & 1.83 RFU & 0.4543 RFU & $-2.149 \mathrm{RFU}$ \\
\hline Top & $96.62 \mathrm{RFU}$ & $101 \mathrm{RFU}$ & 92.86 RFU \\
\hline$K_{d}$ & $560.3 \mathrm{nM}$ & $1.61 \mu \mathrm{M}$ & $6.28 \mathrm{nM}$ \\
\hline HillSlope & -6.857 & 1.12 & 1.301 \\
\hline $\log K_{d}$ & -6.252 & -5.794 & -8.202 \\
\hline Span & 94.79 RFU & 100.5 RFU & 95.01 RFU \\
\hline \multicolumn{4}{|c|}{$95 \% \mathrm{Cl}$ (profile likelihood) } \\
\hline Bottom & -0.8138 to $4.371 \mathrm{RFU}$ & -1.734 to $2.576 \mathrm{RFU}$ & -12.81 to $7.383 \mathrm{RFU}$ \\
\hline Top & 95.11 to $98.96 \mathrm{RFU}$ & 97.36 to $104.9 \mathrm{RFU}$ & 85.12 to $101.4 \mathrm{RFU}$ \\
\hline$K_{d}$ & 543.0 to $576.8 \mathrm{nM}$ & 1.38 to $1.90 \mu \mathrm{M}$ & 4.03 to $1.06 \mathrm{nM}$ \\
\hline HillSlope & $\mathrm{N} / \mathrm{D}^{\alpha}$ & 0.9523 to 1.358 & 0.6759 to 2.567 \\
\hline $\log K_{d}$ & -6.265 to -6.239 & -5.861 to -5.722 & -8.394 to -7.976 \\
\hline \multicolumn{4}{|c|}{ Goodness of Fit } \\
\hline Degrees of Freedom & 26 & 14 & 5 \\
\hline$R$ squared & 0.9947 & 0.9969 & 0.9891 \\
\hline Sum of Squares & 303 & 95.97 & 161.3 \\
\hline Sy.x & 3.414 & 2.618 & 5.68 \\
\hline \multicolumn{4}{|c|}{ Number of points } \\
\hline \# of $X$ values & 30 & 18 & 9 \\
\hline \# Y values analyzed & 30 & 18 & 9 \\
\hline
\end{tabular}


Table S2. Crystallographic statistics.

\begin{tabular}{|c|c|c|c|c|c|}
\hline Structure & L110X Apo & L110X Holo & s112X Holo & W120X Apo & W120X Glycerol \\
\hline \multicolumn{6}{|c|}{ Data collection } \\
\hline Space group & P 1211 & $\mathrm{P} 121$ & I 222 & I 4 & I 41 \\
\hline \multicolumn{6}{|l|}{ Cell dimensions } \\
\hline$a, b, c(\AA)$ & $46.46,85.56,58.10$ & $50.81,98.28,52.65$ & $46.209,93.110,104.369$ & $57.351,57.351,172.320$ & $57.519,57.519,173.026$ \\
\hline$\alpha, \beta, \gamma(\mathrm{deg})$ & $90.000,99.0,90.00$ & $90.00,112.3,90.00$ & $90.000,90.000,90.000$ & $90.000,90.000,90.000$ & 90.00090 .00090 .000 \\
\hline Total Reflections & 614,572 & 340,473 & 36,873 & 956,126 & $2,652,590$ \\
\hline Unique Reflections & 64,975 & 27,711 & 19,561 & 40,032 & 44,646 \\
\hline Resolution $(\AA)$ & $\begin{array}{l}50.00-1.55 \\
(1.58-1.55)\end{array}$ & $\begin{array}{l}50.00-2.10 \\
(2.14-2.10)\end{array}$ & $\begin{array}{l}69.48-1.84 \\
(9.03-1.84)\end{array}$ & $\begin{array}{l}50.00-1.55 \\
(1.58-1.55)\end{array}$ & $\begin{array}{l}50.00-1.50 \\
(1.53-1.50)\end{array}$ \\
\hline $1 / \sigma(I)$ & $10.9(1.3)$ & $13.75(1.9)$ & $16.7(5.9)$ & $32.83(2.36)$ & $36.00(2.14)$ \\
\hline $\mathrm{R}_{\text {meas }}$ & $0.062(0.743)$ & $0.160(0.894)$ & $0.037(0.187)$ & $0.115(1.047)$ & $0.077(1.105)$ \\
\hline $\mathrm{R}_{\text {pim }}$ & $0.030(0.364)$ & $0.076(0.442)$ & $0.026(0.132)$ & $0.036(0.341)$ & $0.021(0.310)$ \\
\hline $\mathrm{CC}_{1 / 2}$ & $(0.938)$ & $.725(0.582)$ & $0.998(0.951)$ & $0.999(0.794)$ & $1.000(0.845)$ \\
\hline $\mathrm{R}_{\text {merge }}$ & -- & -- & $0.026(.132)$ & -- & -- \\
\hline Completeness (\%) & $99.6(93.6)$ & $97.1(92.6)$ & $98.7(99.2)$ & $97.9(99.9)$ & $100.0(100.0)$ \\
\hline Redundancy & $2.1(2.0)$ & $2.2(2.0)$ & $1.9(1.9)$ & $5.2(4.6)$ & $6.8(6.4)$ \\
\hline \multicolumn{6}{|c|}{ Refinement } \\
\hline Resolution $(\AA)$ & $42.78-1.55$ & $49.14-2.10$ & $69.48-1.84$ & $43.08-1.55$ & $29.65-1.50$ \\
\hline $\mathrm{R}_{\text {work }}$ & 0.166 & 0.194 & 0.174 & 0.173 & 0.126 \\
\hline $\mathrm{R}_{\text {free }}$ & 0.201 & 0.256 & 0.212 & 0.197 & 0.167 \\
\hline rmsd bond lengths $(\AA)$ & 0.0221 & 0.0161 & 0.0197 & 0.0245 & 0.0307 \\
\hline rmsd bond angles (deg) & 2.147 & 1.919 & 2.051 & 2.546 & 2.437 \\
\hline
\end{tabular}

*The values in parentheses indicate statistics for the highest resolution shell.

(1) Sano, T.; Pandori, M. W.; Chen, X.; Smith, C. L.; Cantor, C. R. Recombinant Core Streptavidins: A Minimum-Sized Core Streptavidin Has Enhanced Structural Stability and Higher Accessibility to Biotinylated Macromolecules. J. Biol. Chem. 1995, 270 (47), 28204-28209. https://doi.org/10.1074/jbc.270.47.28204.

(2) Wang, J.; Xie, J.; Schultz, P. G. A Genetically Encoded Fluorescent Amino Acid. J. Am. Chem. Soc. 2006, 128 (27), $8738-8739$. https://doi.org/10.1021/ja062666k.

(3) Howarth, M.; Chinnapen, D. J. F.; Gerrow, K.; Dorrestein, P. C.; Grandy, M. R.; Kelleher, N. L.; El-Husseini, A.; Ting, A. Y. A Monovalent Streptavidin with a Single Femtomolar Biotin Binding Site. Nat. Methods 2006, 3 (4), 267-273. https://doi.org/10.1038/nmeth861.

(4) The PyMOL Molecular Graphics System Version 1.8 Schrödinger LLC. The PyMOL Molecular Graphics System, Version 1.8. 2015.

(5) Henderson, J. N.; Simmons, C. R.; Fahmi, N. E.; Jeffs, J. W.; Borges, C. R.; Mills, J. H. Structural Insights into How Protein Environments Tune the Spectroscopic Properties of a Noncanonical Amino Acid Fluorophore. Biochemistry 2020, 59 (37), $3401-3410$. https://doi.org/10.1021/acs.biochem.0c00474.

(6) Moriya, T. Excited-State Reactions of Coumarins in Aqueous Solutions. I. The Phototautomerization of 7-Hydroxycoumarin and Its Derivative. Bull. Chem. Soc. Jpn. 1983, 56 (1), 6-14. https://doi.org/10.1246/bcsj.56.6. 